\title{
A Pilot Study of a Telemedicine-based Substance Use Disorder Evaluation to Enhance Access to Treatment Following Near-Fatal Opioid Overdose
}

\author{
Jeffrey T. Lai \\ Division of Medical Toxicology \\ Department of Emergency Medicine \\ University of Massachusetts Medical \\ School, Worcester, MA, USA \\ jeffrey.lai@umassmemorial.org \\ Amy D. Costigan \\ Department of Emergency Medicine \\ University of Massachusetts Medical \\ School, Worcester, MA, USA \\ amy.costigan@umassmemorial.org
}

\author{
Brittany P. Chapman \\ Division of Medical Toxicology \\ Department of Emergency Medicine \\ University of Massachusetts Medical \\ School, Worcester, MA, USA \\ brittany.chapman@umassmed.edu
}

Karla M. Rodriguez-Perez

Department of Psychiatry

University of Massachusetts Medical

School, Worcester, MA, USA

karla.rodriguez@umassmemorial.org

Kavita M. Babu

Division of Medical Toxicology

Department of Emergency Medicine

University of Massachusetts Medical

School, Worcester, MA, USA

kavita.babu@umassmemorial.org

\author{
Stephanie P. Carreiro \\ Division of Medical Toxicology \\ Department of Emergency Medicine \\ University of Massachusetts Medical \\ School, Worcester, MA, USA \\ stephanie.carreiro@umassmed.edu
}

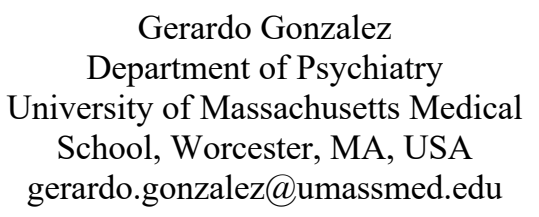

Gerardo Gonzalez

School, Worcester, MA, USA

gerardo.gonzalez@umassmed.edu

\begin{abstract}
The opioid epidemic is a growing public health emergency in the United States, with deaths from opioid overdose having increased five-fold since 1999. Emergency departments (EDs) are the primary sites of medical care after near-fatal opioid overdose but are poorly equipped to provide adequate substance use treatment planning prior to discharge. In many underserved locales, limited access to clinicians trained in addiction medicine and behavioral health exacerbates this disparity. In an effort to improve postoverdose care in the ED, we developed a telemedicine protocol to facilitate timely access to substance use disorder evaluations. In this paper, we describe the conception and refinement of the telemedicine program, our experience with the first 20 participants, and potential implications of the platform on health disparities for individuals with opioid use disorder.
\end{abstract}

\section{Introduction}

The death toll from the opioid epidemic continues to rise, with 47,600 individuals in the United States of
America dying from opioid overdose in 2017, a 43.8\% increase from 2015 [1,2]. Emergency departments (EDs) represent the primary sites of medical care after opioid overdose, but often are ill-equipped to provide adequate substance use treatment referrals prior to a patient's discharge. This results in a cycle of successive resuscitations from overdose followed by discharges, often culminating in a final fatal overdose $[3,4]$.

Disparities in access to healthcare, health behaviors, and health outcomes are well documented between populations in rural areas and their urban counterparts [5-7]. These differences persist even when considered independently of race/ethnicity and socioeconomic status [8]. People who use drugs (PWUD) are another medically underserved population that is at risk for adverse health outcomes [9-11]. The subset of PWUD residing outside of major urban centers thus represents a particularly high-risk group at a significant health disadvantage [11, 12]. Therefore, there is a critical need to address the health inequity that leads to disproportionate morbidity and mortality risk among PWUD in rural areas.

To address the opioid epidemic, the state of Massachusetts enacted legislation in 2016 mandating that individuals presenting to the ED after suspected 
opioid overdose be offered a Substance Use Disorder Evaluation (SUDE) [13]. This targeted, 20-minute intervention conducted by a behavioral health clinician is adapted from the evidence-based Screening, Brief Interview and Referral to Treatment (SBIRT), and identifies individual patterns of substance use, details appropriate level of addiction care, and motivates patients to seek treatment [13, 14]. As EDs in Massachusetts and in other states seek to improve the care they provide to PWUD, they face a critical limitation in that a qualified clinician trained in the treatment of opioid use disorder may not be available in a timely fashion during the post-overdose period [15]. This issue is exacerbated in rural and underserved areas.

Ideally, an individual who is assessed in the ED for opioid overdose would have ready access to a behavioral health clinician who can help devise a collaborative long-term treatment plan. However, the limited availability of addiction medicine resources often results in unintended delays that can lead a frustrated patient to decline a SUDE and leave the hospital without so much as a discussion of evidencebased therapies such as MAT (e.g., opioid agonist therapy with methadone or partial agonist therapy with buprenorphine or Suboxone $\left.{ }^{\circledR}\right)[16,17]$. This is a critical missed opportunity; individuals who are successfully revived with naloxone following an opioid overdose are at high risk of death within the subsequent year [18]. Therefore, the ED visit after a near-fatal overdose represents an opportunity to bridge this vulnerable population to long-term treatment; a failure to intervene during this critical time carries significant mortality risk.

Telemedicine represents a promising solution to these missed opportunities. A recent review found that telemedicine may have equivalent outcomes as compared to in-person services for indications such as mental health and rehabilitation [19]. However, the application of telemedicine specifically to substance use disorder treatment is not as well studied; one study found that utilization rates appear to be increasing, but overall remain very low [20]. Prior work has characterized the use of telemedicine by organizations that provide outpatient care for substance use disorder as being primarily in the form of computerized screening programs, telephone-based recovery support systems, and telephone-based therapy [21]. There is demonstrated interest from addiction medicine clinicians in developing videoconferencing for the provision of substance use disorder treatment, but there are anticipated barriers to implementation, including concerns of startup costs, lack of reimbursement for services, unfamiliarity with technology, inexperience with telemedicine, and confidentiality concerns [22].

Telemedicine in the ED setting is perhaps most familiar in the form of videoconferencing for timesensitive neurologic emergencies, such as suspected acute cerebrovascular accidents (strokes) [23]. In the present study, we sought to extend the impact of EDbased telemedicine to the treatment of opioid use disorder by developing a telemedicine-based substance use disorder evaluation (teleSUDE) platform, and to gather preliminary data regarding its feasibility and usability. The ultimate goal of teleSUDE is to provide immediate, timely access to an addiction medicine clinician in EDs where an inperson evaluation may not be logistically possible.

\section{TeleSUDE technology platform}

We envisioned teleSUDE as a system that would be familiar, intuitive, and easy to use for both clinician and patient. To this end, we obtained a pair of matched Apple iPad Pro tablet computers (San Jose, CA, USA) equipped with Zoom (San Jose, CA, USA), a Health Insurance Portability and Accountability Act (HIPAA)-compliant software backbone that is device and operating system agnostic.

The tablet computer was secured to a JACO mobile tablet cart equipped with a battery pack (Franklin, MA, USA) (Figure 1). When a teleSUDE clinician activates Zoom, an encrypted video conference call similar to "FaceTime" was made to the paired tablet computer accessed by the patient (Figure 2). A split screen allowed clinicians and patients to see each other in real-time throughout the duration of the telemedicine encounter (Figure 3).

The SUDE clinician was given a standardized 20minute training on how to operate teleSUDE as well as troubleshoot issues with connectivity, potential loss of video and voice streams, and techniques to reestablish the teleSUDE in the instance of lost connectivity. The clinician demonstrated competency operating teleSUDE by completing mock evaluations with trained members of the study team.

\section{Design considerations}

To enhance portability in a busy ED environment, we elected to use a pair of matched tablet computers equipped with WiFi connectivity as the backbone for our teleSUDE platform. We sought to maximize usability and minimize startup costs by utilizing existing consumer-grade tablet computers, rather than purchasing specialized medical teleconferencing equipment, which is often unwieldy and expensive. 


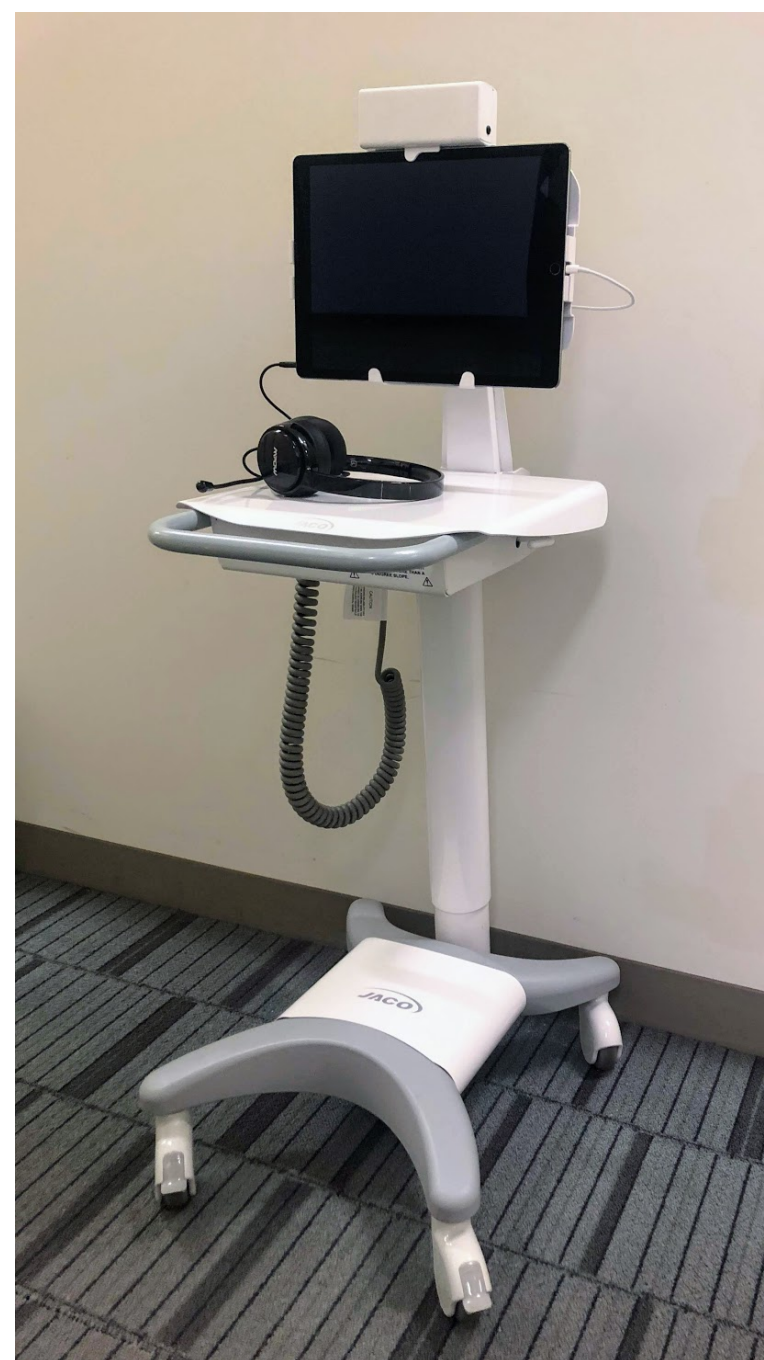

Figure 1. TeleSUDE apparatus consisting of an Apple iPad Pro tablet computer mounted on a locking JACO mobile tablet cart.

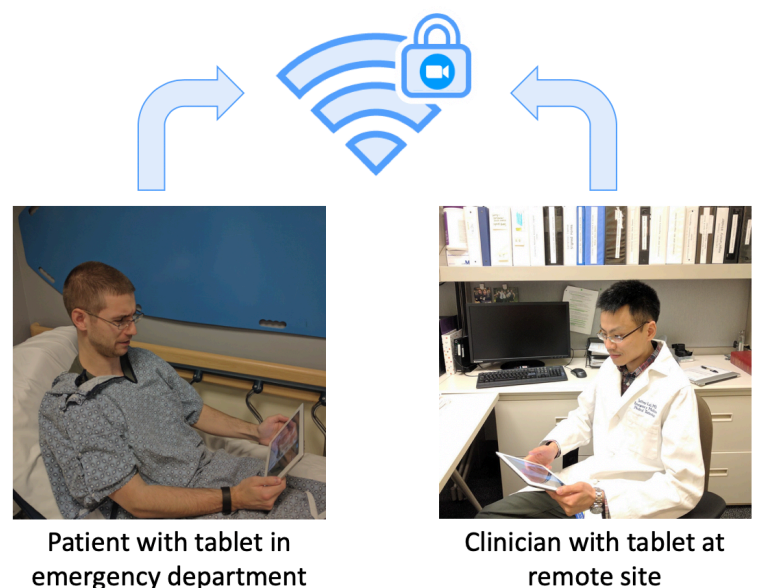

Figure 2. Schematic representation of teleSUDE protocol.

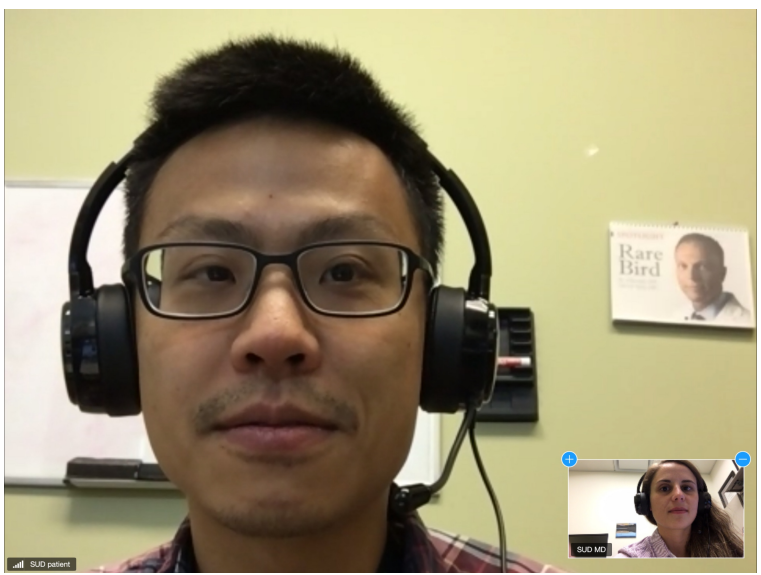

Figure 3. Screen capture demonstrating the functionality of the teleSUDE protocol during a simulated encounter between study staff.

We selected Apple tablet computers over other similar devices because of their ubiquity and uniform user interface, as well as predictable product support cycles. We felt that Apple's iOS offered a distinct advantage over other operating systems, which can suffer from version fragmentation and inconsistent user experiences due to proprietary graphic user interface overlays.

By using a telemedicine suite that is device and operating system agnostic, teleSUDE will have maximum flexibility and scalability, as it will not be limited to a hospital's specific computer systems. The only requirement for delivering teleSUDE will be a WiFi connection, which is ubiquitous in modern hospitals.

Initially, we had intended to use the tablet computer's integrated speaker and microphone for participant encounters in a similar fashion to devices currently in use at our institution for acute neurologic emergencies, however it became apparent based on early feedback from the teleSUDE clinician and study participants that incorporating over-the-ear headphones with a microphone may be a better solution. This had the enhanced benefit of ensuring an additional level of privacy and protection of sensitive health information, which may encourage patients to be more candid regarding their substance use.

\section{Methods}

Prior to initiation of the study protocol, teleSUDE was assembled and deployed in our ED using mock encounters with study staff. Field testing was performed by study staff using paired tablet computers within various patient care areas at our institution, with attention to connectivity in areas where interference 
may arise (e.g. in the vicinity of cardiac telemetry monitors, diagnostic radiology studies, cellular phone use by patients and clinicians). Once we confirmed functionality, we initiated study enrollment.

This was a single arm, prospective observational study approved by the University of Massachusetts Medical School Institutional Review Board. A convenience sample of participants presenting to the ED after a known or suspected opioid overdose was utilized. Participants were eligible for the study if they received naloxone (the antidote for opioid overdose) in the pre-hospital or ED setting, were 18 years of age or older, English-speaking, and able to provide informed consent. Potential subjects were excluded if they were pregnant, prisoners or in police custody, or presenting with a primary psychiatric complaint.

Once a potential participant was deemed eligible, they were approached and enrolled by a member of the study team. Individuals who declined to participate received the standard of care, an in-person encounter with a SUDE clinician. Participants who consented were given a brief overview of the teleSUDE platform and provided with the opportunity to ask questions. Participants then received their SUDE via the telemedicine interface.

At the conclusion of the telemedicine encounter, study staff administered a brief survey based on the technology acceptance model framework regarding participant attitudes toward and acceptance of the technology artifact [24]. Study staff then probed further regarding the participant's overall experience. Once the participant encounter was completed, study staff prepared a debrief summarizing key points that arose during the intervention. Study staff also followed up with the teleSUDE clinician to obtain feedback regarding their overall perception on how the encounter went and if any technical errors arose.

\section{Results}

\subsection{Participant characteristics}

Twenty-seven individuals were approached to participate. Twenty consented to participate in our pilot study, and seven declined. Median age was 32 years, 13 participants identified as male, and 15 participants identified as Caucasian. Demographic characteristics of participants can be found in Table 1 . The individuals who declined to participate cited a general dislike of technology $(\mathrm{N}=2)$, a lack of interest in receiving a SUDE $(\mathrm{N}=1)$, and an unwillingness to partake in a research study $(\mathrm{N}=4)$. Nineteen of the 20 participants who consented completed their SUDE via telemedicine. The remaining individual consented to participate, but was unable to complete the study protocol because the tablet computer's battery was depleted.

\section{Table 1. Demographic characteristics of participants.}

\begin{tabular}{|l|l|}
\hline & $\mathrm{N}=20$ \\
\hline Median age, years (IQR) & $32(30,48)$ \\
\hline & \\
\hline Sex & \\
\hline Male & 13 \\
\hline Female & 7 \\
\hline Ethnicity/Race & \\
\hline White, non-Hispanic & 15 \\
\hline Black, non-Hispanic & 1 \\
\hline Hispanic & 2 \\
\hline Other & 2 \\
\hline
\end{tabular}

\subsection{Participant perceptions of usability and acceptability}

Sixteen participants responded to the postencounter survey regarding teleSUDE's usability and acceptability (Table 2). Unfortunately, three participants did not complete the survey for the following reasons: time-sensitive requirements of another research study (1), significant restlessness and inability to concentrate (1), and survey responses failing to sync properly (1). However, study staff did record informal notes regarding these three participants' attitudes toward teleSUDE.

The technology was favorably reviewed by all study participants, with two subjects describing the technology artifact as "cool" and one going so far as to state that teleSUDE is the "best thing since sliced bread and bacon, and I'm a Georgia boy so I love bacon." Participants were unanimous in rating the technology as easy to use and found the audio and video quality to be satisfactory, despite two participants describing pre-existing "hearing problems" and one stating they were "legally blind without glasses." Three participants stated that they would prefer telemedicine over an in-person encounter, including one who admitted they were "not a tech person" but cited teleSUDE's advantages as being "quick, convenient, and private." Three participants commented that they would prefer an inperson encounter, though they still found the telemedicine encounter satisfactory. The majority reported that they thought teleSUDE felt similar to having an in-person encounter. Two participants noted that the location of the camera on the tablet computer (to the left of the screen rather than above the screen in the midline) gave the appearance that the clinician 
was looking elsewhere during the encounter, and that this made the encounter feel less personal than it otherwise could have been.

Table 2. Participant responses to usability and acceptability survey.

\begin{tabular}{|l|l|l|}
\hline Characteristic (N=16) & $\begin{array}{l}\text { Agree } \\
\text { No. (\%) }\end{array}$ & $\begin{array}{l}\text { Strongly } \\
\text { Agree } \\
\text { No. (\%) }\end{array}$ \\
\hline $\begin{array}{l}\text { Amenable to having SUDE } \\
\text { via telemedicine }\end{array}$ & $6(38)$ & $10(62)$ \\
\hline TeleSUDE was easy to use & $7(44)$ & $9(56)$ \\
\hline $\begin{array}{l}\text { Interacting with clinician } \\
\text { via telemedicine felt similar } \\
\text { to an in-person interaction }\end{array}$ & $7(44)$ & $8(50)$ \\
\hline $\begin{array}{l}\text { Quality of audio was } \\
\text { satisfactory }\end{array}$ & $4(27)$ & $11(73)$ \\
\hline $\begin{array}{l}\text { Quality of video was } \\
\text { satisfactory }\end{array}$ & $9(56)$ & $7(44)$ \\
\hline $\begin{array}{l}\text { Felt assured that the } \\
\text { assessment was confidential }\end{array}$ & $6(38)$ & $10(62)$ \\
\hline
\end{tabular}

\subsection{Clinician feedback}

The teleSUDE clinician reported that the technology was easy to use and that interacting with participants through telemedicine was similar to evaluating patients in person. The clinician did note significant ambient noise during three encounters, with the noise level becoming a distraction in two of these. This was mitigated in subsequent encounters by the use of over-ear headphones equipped with directional microphones, which markedly decreased transmission of noise from adjacent care areas.

\subsection{Technology-related issues}

A major technical failure occurred during one of the encounters when a participant who consented to the study protocol was unable to complete the telemedicine encounter because the tablet computer's battery had not been recharged and was fully depleted.

Minor technical difficulties were identified during three $(15 \%)$ of the encounters. These consisted of brief latency issues with the video stream that both parties felt were insignificant and did not impact the flow of the evaluation. During another encounter, the video feed of the clinician failed to display on the participant's tablet; this was quickly resolved by terminating the session and initiating a new one. Overall, the quality of the video conference was deemed satisfactory during all completed encounters.

\section{Discussion}

In this investigation, we gathered formative evidence that a low-intensity, affordable telemedicine platform is a feasible and acceptable way to conduct sensitive substance use disorder evaluations in the emergency department. Participants were able to operate the technology, and we were able to efficiently train a clinician to use teleSUDE. These data are significant because they demonstrate that first, patients who have overdosed on opioids are accepting of a telemedicine platform to conduct SBIRT evaluations, and second, the technology platform is a viable solution in the dynamic emergency department environment.

While telemedicine has been used previously to provide psychiatric evaluations, this study represents the first time that it has been leveraged to provide substance use disorder evaluations for patients in the ED after near-fatal overdose [25, 26]. Participants were agreeable to the use of telemedicine, and most found it equivalent (or in some cases preferable) to standard in-person evaluations. This finding is reflected in other ED-based telemedicine programs, such as a study from our institution using Google Glass technology to provide remote dermatology consultation in the ED [27].

We experienced only one major technical failure during our pilot, in which the telemedicine encounter could not be completed because the tablet computer's battery was depleted. We subsequently modified our study protocol to ensure that all devices would be adequately recharged following use. Otherwise, we found that technological difficulties were generally minor and centered around transmission of ambient noise as well as brief network interruptions. We found that the first was easily remedied by utilizing headphones with directional microphones and that the second was essentially a non-issue.

Given the societal stigma that persists regarding substance use disorder, PWUD understandably have significant concerns regarding preservation of privacy. Indeed, this has been demonstrated in rural areas with small close-knit communities, where individuals have reported not seeking care at treatment centers because their entire town will then be aware of their substance use disorder [28]. Our participants reported feeling assured that the teleSUDE assessment was private and confidential. This finding suggests that telemedicine may represent a unique solution to this issue: utilizing an encrypted HIPAA-compliant videoconferencing suite may allow PWUD in rural environments to access care for opioid use disorder in a secure and discreet manner. 
This pilot program demonstrates that teleSUDE can be used to enhance the availability of substance use disorder counseling and treatment in patients presenting for evaluation after near-fatal overdose. Engaging PWUD to understand factors leading to acceptance or non-acceptance of telemedicine interventions is essential to the development of a scalable platform for providing addiction care resources.

\subsection{Limitations}

The limitations of our small pilot study are as follows. The study was aimed at assessing the feasibility and acceptability of using a telemedicine platform to deliver SUDEs to PWUD in a single highvolume ED. The opinions of our limited number of participants may not be generalizable to the population of PWUD at large. Additionally, the resources available and the clinical environment at our academic tertiary care hospital are unlikely to be representative of the settings where teleSUDE would ultimately be utilized (e.g. community hospitals or rural critical access hospitals). However, this preliminary phase study is crucial to determining if PWUD will accept the technology before deploying this platform to the ultimate target users. Furthermore, once feasibility and acceptability are established, a suitable billing and reimbursement model must be developed in order to provide this vital service to communities in need in an affordable, sustainable fashion.

\subsection{How teleSUDE can be utilized to overcome health disparities}

Lack of funding and difficulties with transportation have been identified as barriers to improving addiction care in both urban and rural settings [29]. However, these impediments are particularly problematic in rural settings, where the populace tends to be less affluent and more geographically dispersed, and specialized resources for individuals with co-morbid conditions such as human immunodeficiency virus (HIV) or psychiatric illnesses are scarce [29, 30]. Substance use treatment clinicians in rural areas report that the scarcity of public transportation combined with individuals residing far away from available resources creates a scenario where people seeking treatment must rely on friends or family members for transportation, some of whom have been "enabling or using with them," leading to "additional, more severe, and more persistent transportation challenges" [29].

Telemedicine is currently being studied as a means of creating access to mental health services for individuals afflicted by bipolar disorder and posttraumatic stress disorder residing in rural communities where specialist care is unavailable [31]. Similarly, teleSUDE seeks to leverage advances in telemedicine to bring the expertise of substance use treatment specialists based at a tertiary-care academic medical center out to our rural communities where some of the most disadvantaged populations reside. TeleSUDE can ameliorate infrastructure deficiencies by allowing PWUD to receive counseling and evaluations within the comfort of their residence, obviating the potentially costly and time-consuming need to find transportation to a distant locale. Additionally, teleSUDE clinicians can serve as the centralized contact point, helping PWUD in rural areas navigate the complexities of the healthcare system and coordinating care among the various aspects of substance use treatment (e.g., establishing care with community-based substance use counseling programs, finding openings in supervised detoxification centers, and assisting in referrals for the management of cooccurring medical and psychiatric conditions).

The overarching goal of teleSUDE is to decrease morbidity and mortality from opioid overdose by providing a critical intervention during the immediate post-overdose period for PWUD residing in lowresource communities. Historically, this population is disproportionately underserved by the healthcare system. TeleSUDE will help to level the playing field for these individuals, bringing evidence-based addiction care across time and distance to areas where it is needed most.

\subsection{Future directions}

Medications for addiction treatment (MAT), including buprenorphine and methadone, represent the current evidence-based standard of care for the treatment of opioid use disorder. Donofrio et al showed that initiation of buprenorphine therapy by a qualified emergency medicine provider with a plan to transition care to an addiction center is more effective than behavioral counseling alone in the immediate post-overdose period [32]. However ED initiation of MAT requires coordination of several resources, including licensed providers with a special training certification ("X-waiver"), and requires a concerted effort even in resource rich environments. While further study is necessary to demonstrate the generalizability of this approach, ED-initiation of MAT via teleSUDE may represent a major breakthrough in ED-based post-overdose care, as many communities and rural EDs lack providers with the requisite certifications to prescribe buprenorphine [33]. 
The next step in our work is to use teleSUDE to not only link PWUD in underserved areas to addiction medicine clinicians, but also to facilitate immediate access to MAT with a concrete plan for downstream linkage to long-term addiction care. By connecting post-overdose patients in rural EDs with specialized substance use disorder providers located at a remote site, teleSUDE will enhance the ability of EDs to perform "warm handoffs" to outpatient substance use treatment centers and increase access to much needed addiction care resources during the vulnerable postoverdose period. We envision a world in which PWUD in rural Appalachia can receive the same highquality addiction care as PWUD in New York City.

\section{Conclusions}

Our preliminary data suggest that telemedicine delivery of substance use disorder evaluations is acceptable to patients and intuitive for providers. TeleSUDE can enhance access to addiction treatment programs and facilitate the efficient and effective delivery of evidence-based post-overdose care, including ED-based initiation of MAT. Our technology platform represents an innovative method of delivering treatment for opioid use disorder during the critical post-overdose period, and holds immense potential for improving access to addiction care for PWUD who reside in underserved areas.

\section{Acknowledgments}

This research was supported by the University of Massachusetts (UMass) Memorial Medical Group Prize for Academic Collaboration and Excellence, the UMass Memorial Medical Center Virtual Medicine Program, the UMass Emergency Medicine Research Fund, NIDA K23DA045242 (SPC), and the Medical Toxicology Foundation Practice Award (JTL).

We thank Drs. Peter Chai and Edwin Boudreaux for their insightful discussion and feedback during the early stages of this work, and Nicole Day for her tireless dedication to improving post-overdose care for PWUD.

\section{References}

[1] L. Scholl, P. Seth, M. Kariisa, N. Wilson, and G. Baldwin, "Drug and Opioid-Involved Overdose Deaths United States, 2013-2017," MMWR Morb Mortal Wkly Rep, vol. 67, no. 5152, pp. 1419-1427, Jan 4 2018, doi: 10.15585/mmwr.mm675152e1.

[2] C. f. D. C. a. Prevention, "Annual Surveillance Report of Drug-Related Risks and Outcomes - United States, 2017,"
August 31, 2017 2017. Accessed: April 24, 2018. [Online]. Available:

https://www.cdc.gov/drugoverdose/pdf/pubs/2017-cdcdrug-surveillance-report.pdf

[3] A. Uusküla et al., "Non-fatal overdoses and related risk factors among people who inject drugs in St. Petersburg, Russia and Kohtla-Järve, Estonia," (in eng), BMC Public Health, vol. 15, pp. 1255-1255, 2015, doi: 10.1186/s12889015-2604-6.

[4] G. W. Miller, "R.I. opioid crisis: After son\&apos;s death, they vow to change system," Providence Journal. [Online]. Available:

http://www.providencejournal.com/news/20160319/riopioid-crisis-after-sons-death-they-vow-to-change-system.

[5] T. J. Anderson, D. M. Saman, M. S. Lipsky, and M. N. Lutfiyya, "A cross-sectional study on health differences between rural and non-rural U.S. counties using the County Health Rankings.," (in English), BMC health services research, vol. 15, p. 441, Oct 2015, doi: 10.1186/s12913015-1053-3.

[6] C. A. Befort, N. Nazir, and M. G. Perri, "Prevalence of obesity among adults from rural and urban areas of the United States: findings from NHANES (2005-2008)." (in English), The Journal of rural health : official journal of the American Rural Health Association and the National Rural Health Care Association, vol. 28, no. 4, pp. 392-397, 2012, doi: 10.1111/j.1748-0361.2012.00411.x.

[7] M. E. Roberts et al., "Rural tobacco use across the United States: How rural and urban areas differ, broken down by census regions and divisions.," (in English), Health \& place, vol. 39, pp. 153-159, May 2016, doi: 10.1016/j.healthplace.2016.04.001.

[8] J. T. Caldwell, C. L. Ford, S. P. Wallace, M. C. Wang, and L. M. Takahashi, "Intersection of Living in a Rural Versus Urban Area and Race/Ethnicity in Explaining Access to Health Care in the United States.," (in English), American journal of public health, vol. 106, no. 8, pp. 1463-1469, Aug 2016, doi: 10.2105/AJPH.2016.303212.

[9] D. Ciccarone et al., "Soft tissue infections among injection drug users--San Francisco, California, 19962000.," (in English), MMWR. Morbidity and mortality weekly report, vol. 50, no. 19, pp. 381-384, May 2001. [Online]. Available: http://eutils.ncbi.nlm.nih.gov/entrez/eutils/elink.fcgi?dbfro $\underline{\mathrm{m}=\text { pubmed\&amp;id=11465906\&amp;retmode }=\text { ref\&amp;c }}$ md=prlinks.

[10] A. Kamarulzaman and F. L. Altice, "Challenges in managing HIV in people who use drugs.," (in English), Current opinion in infectious diseases, vol. 28, no. 1, pp. 1016, Feb 2015, doi: 10.1097/QCO.0000000000000125.

[11] I.-M. Chen, C. L.-C. Huang, B.-J. Yeh, and Y.-L. Chien, "Health service utilization of heroin abusers: a retrospective 
cohort study.," (in English), Addictive Behaviors, vol. 45, pp. 281-286, Jun 2015, doi: 10.1016/j.addbeh.2015.01.042.

[12] R. A. Crosby, C. B. Oser, C. G. Leukefeld, J. R. Havens, and A. Young, "Prevalence of HIV and risky sexual behaviors among rural drug users: does age matter?," (in English), Annals of epidemiology, vol. 22, no. 11, pp. 778782, Nov 2012, doi: 10.1016/j.annepidem.2012.07.006.

[13] C. o. Massachusetts. "Chapter 52 - Acts of 2016." http://www.massmed.org/opioidbill2016/\#.WHcK37GZOY $\underline{\text { V }}$ (accessed January 27, 2017).

[14] B. K. Madras, W. M. Compton, D. Avula, T. Stegbauer, J. B. Stein, and H. W. Clark, "Screening, brief interventions, referral to treatment (SBIRT) for illicit drug and alcohol use at multiple healthcare sites: Comparison at intake and 6 months later," (in English), Drug and Alcohol Dependence, vol. 99, no. 1-3, pp. 280-295, Jan 2009, doi: 10.1016/j.drugalcdep.2008.08.003.

[15] C. Vestal, "Facing an overdose epidemic, some ERs now offer addiction treatment," in The Washington Post, ed, 2018.

[16] A. Hanly et al., "The Path Forward: Substance Use Disorder Evaluations (SUDE) in the Emergency Department," Journal of Medical Toxicology, vol. 14, no. 1, pp. 3-67, 2018. [Online]. Available: https://doi.org/10.1007/s13181-018-0655-7.

[17] L. Gowing, M. F. Farrell, R. Bornemann, L. E. Sullivan, and R. Ali, "Oral substitution treatment of injecting opioid users for prevention of HIV infection.," (in English), The Cochrane database of systematic reviews, no. 8, p. CD004145, Aug 2011, doi: 10.1002/14651858.CD004145.pub4.

[18] S. G. Weiner, O. Baker, D. Bernson, and J. D. Schuur, "One-Year Mortality of Patients After Emergency Department Treatment for Nonfatal Opioid Overdose," Annals of emergency medicine, 2019. [Online]. Available: http://www.sciencedirect.com/science/article/pii/S0196064 419303439.

[19] E. Shigekawa, M. Fix, G. Corbett, D. H. Roby, and J. Coffman, "The Current State Of Telehealth Evidence: A Rapid Review," (in English), Health Affairs, vol. 37, no. 12, pp. 1975-1982, Dec 2018, doi: 10.1377/hlthaff.2018.05132.

[20] H. A. Huskamp et al., "How Is Telemedicine Being Used In Opioid And Other Substance Use Disorder Treatment?," (in English), Health Affairs, vol. 37, no. 12, pp. 1940-1947, Dec 2018, doi: 10.1377/hlthaff.2018.05134.

[21] T. Molfenter, R. Brown, A. O’Neill, E. Kopetsky, and A. Toy, "Use of Telemedicine in Addiction Treatment: Current Practices and Organizational Implementation Characteristics," (in English), International Journal of Telemedicine and Applications, vol. 2018, no. 8, pp. 1-7, 2018, doi: 10.1155/2018/3932643.
[22] T. Molfenter, M. Boyle, D. Holloway, and J. Zwick, "Trends in telemedicine use in addiction treatment," Addiction Science \& Clinical Practice, vol. 10, no. 1, p. 14, 2015. [Online]. Available: https://doi.org/10.1186/s13722015-0035-4.

[23] N. Chalouhi et al., "Intravenous tissue plasminogen activator administration in community hospitals facilitated by telestroke service.," (in English), Neurosurgery, vol. 73, no. 4, pp. 667-71- discussion 671-2, Oct 2013, doi: 10.1227/NEU.0000000000000073.

[24] R. J. Holden and B.-T. Karsh, "The Technology Acceptance Model: Its past and its future in health care," Journal of Biomedical Informatics, vol. 43, no. 1, pp. 159172, 2010/02/01/ 2010, doi: https://doi.org/10.1016/j.jbi.2009.07.002.

[25] M. B. Richard O'Reilly , F.R.C.P.C. , M. D. Joan Bishop, F.R.C.P.C. , R. N. Karen Maddox , M.A. , M. D. Lois Hutchinson, F.R.C.P.C. , M. B. Michael Fisman, F.R.C.P.C. , and M. D. Jatinder Takhar , F.R.C.P.C. „, "Is Telepsychiatry Equivalent to Face-to-Face Psychiatry? Results From a Randomized Controlled Equivalence Trial," Psychiatric Services, vol. 58, no. 6, pp. 836-843, 2007, doi: 10.1176/ps.2007.58.6.836.

[26] I. Modai, M. Jabarin, R. Kurs, P. Barak, I. Hanan, and L. Kitain, "Cost effectiveness, safety, and satisfaction with video telepsychiatry versus face-to-face care in ambulatory settings.," (in English), Telemedicine journal and e-health : the official journal of the American Telemedicine Association, Comparative Study vol. 12, no. 5, pp. 515-520, Oct 2006, doi: 10.1089/tmj.2006.12.515.

[27] P. R. Chai et al., "Feasibility and Acceptability of Google Glass for Emergency Department Dermatology Consultations.," (in English), JAMA dermatology, vol. 151, no. 7, pp. 794-796, Jul 2015, doi: 10.1001/jamadermatol.2015.0248.

[28] R. L. Sexton, R. G. Carlson, C. G. Leukefeld, and B. M. Booth, "Barriers to formal drug abuse treatment in the rural south: a preliminary ethnographic assessment.," (in English), Journal of psychoactive drugs, vol. 40, no. 2, pp. 121-129, Jun 2008, doi: 10.1080/02791072.2008.10400621.

[29] E. Pullen and C. Oser, "Barriers to substance abuse treatment in rural and urban communities: counselor perspectives.," (in English), Substance Use \&amp; Misuse, vol. 49, no. 7, pp. 891-901, Jun 2014, doi: $10.3109 / 10826084.2014 .891615$.

[30] J. L. Pringle, N. P. Emptage, and R. L. Hubbard, "Unmet needs for comprehensive services in outpatient addiction treatment.," (in English), Journal of substance abuse treatment, vol. 30, no. 3, pp. 183-189, Apr 2006, doi: 10.1016/j.jsat.2005.11.006. 
[31] P.-C. O. R. Institute. "Comparing Two Approaches to Provide Complex Mental Health Care for Patients in Rural Areas -- $\quad$ The SPIRIT Study." https://www.pcori.org/research-results/2015/comparingtwo-approaches-provide-complex-mental-health-carepatients-rural (accessed August 30, 2019).

[32] G. D’Onofrio et al., "Emergency Department-Initiated Buprenorphine/Naloxone Treatment for Opioid Dependence," (in English), JAMA, vol. 313, no. 16, pp. 1636-9, Apr 28 2015, doi: 10.1001/jama.2015.3474.

[33] L. E. Sullivan and D. A. Fiellin, "Office-Based Buprenorphine for Patients with Opioid Dependence," Annals of internal medicine, vol. 148, no. 9, pp. 662-670, May $062008 . \quad$ [Online]. Available: http://www.ncbi.nlm.nih.gov/pmc/articles/PMC3694223/. 